\title{
Lattice Defects in Deformed Low Carbon Steels and the Annealing Stage*
}

\author{
By Jin-ichi TAKAMURA, ${ }^{* *}$ Isao TAKAHASHI, ${ }^{* * *}$ and Muneyuki AMANO ${ }^{* * * * * *}$
}

\begin{abstract}
Synopsis
Successive two annealing stages corresponding to the annihilaiion of dislocations in deformed low carbon steels were found from electrical resistivity measurements. For example, on $5 \mathrm{~min} / 20^{\circ} \mathrm{C}$ isochronal annealing of a $0.03 \%$ carbon steel stretched by $20 \%$ at $-75^{\circ} \mathrm{C}$, the two stages were centered around $600^{\circ}$ and $680^{\circ} \mathrm{C}$. The former stage was associated with the formation of sub-boundaries, and the latter with the recrystallization. The fact was confirmed by the measurement of yield stress and also by transmission electron microscopy. The similar stages were also seen for high purity iron, but the central temperatures of the stages were lower than those in carbon steels.

The dislocation density and the concentration of point defects produced by plastic deformation were determined from the resistivity measurements, and the results showed that the amount of lattice defects, and in particular the density of dislocations, increases as the concentration of solute carbon is increased and also as the grain size of specimen is reduced.
\end{abstract}

\section{Introduction}

Annealing experiments of lattice defects produced by plastic deformation in iron have been made by a number of investigators, ${ }^{1)-4}$ ) but in these studies attention has been paid mostly to annealing stages associated with point defects. Cuddy ${ }^{2}$ has reported that the strain-induced resistivity in pure iron decreases monotonously over the temperature range from $-196^{\circ}$ to $500^{\circ} \mathrm{C}$ with no indication of any uniquely activated process, and he has concluded that at least a part of the low temperature recovery is related to the rearrangement of dislocations to relax their strain fields.

In this sort of experiments, however, it is very difficult to separate the resistivity change directly connected with lattice defects from that related to residual interstitial impurities such as carbon or nitrogen. ${ }^{5)}$ In low carbon steels, in particular, these impurities give rise to large resistivity change on annealing, and the stage due to the annihilation of dislocations has never been reported from resistivity measurements.

The present investigation aims primarily at determining the density of dislocations introduced by plastic deformation in low carbon steels from the electrical resistivity change at the annealing stage that corresponds to the annihilation of dislocations. Once the dislocation density is determined, the concentration of point defects can also be obtained by subtracting the resistivity change due to the dislocation annihilation from the total resistivity increment due to straining. For comparison with low carbon steels a high purity iron having the carbon content less than 1 ppm was used, and the effect of solute carbon on the production of lattice defects by plastic deformation was studied.

\section{Experimental Procedure}

Materials mainly used are vacuum-melted irons containing $0.03 \%$ carbon (\#E), $0.01 \%$ carbon $(\# \mathrm{~F})$, and $0.002 \%$ carbon (\#A), as listed in Table 1. Polycrystalline wires of $1.0 \mathrm{~mm}$ dia were used for electrical resistivity measurements and also for tensile testing, and plates $1.0 \mathrm{~mm}$ in thickness for transmission electron microscopy.

As the reference material a high purity iron, which was purified from the Johnson-Matthey \& Co. pure iron (\#JM as-received) by a special method as described below, was used (\#JM purified). The chemical compositions of \#JM as-received are also in Table 1. In the purification method, wires of $1.0 \mathrm{~mm}$ dia drawn from \# JM rods were subjected to annealing at about $600^{\circ} \mathrm{C}$ for a few hundred hours in low vacuum of about $10^{-1}$ torr. with the subsequent annealing in palladium-purified hydrogen at temperatures below $500^{\circ} \mathrm{C}$ for more than twenty hours. By this treatment the ratio of the resistivity at $25^{\circ} \mathrm{C}$ to that at liquid nitrogen temperature, $\rho_{298} / \rho_{77}$, was increased from 9 (\#JM as-received) to $17.0 \sim 17.3$ (\#JM purified). The specimen having a ratio $\rho_{298} / \rho_{77}=17.3$ showed resistivity ratio $\rho_{298} / \rho_{4 \cdot 2}$ to be $215 .^{6)}$ The total amount of interstitial impurities such as carbon and nitrogen in

Table 1. Chemical compositions of materials (wt \%)

\begin{tabular}{|c|c|c|c|c|c|c|c|}
\hline Specimen & $\mathrm{C}$ & $\mathrm{N}$ & $\mathrm{O}$ & $\mathrm{Si}$ & $\mathrm{Mn}$ & $\mathrm{P}$ & $\mathrm{S}$ \\
\hline$\# A$ & 0.002 & 0.0041 & 0.0176 & $<0.001$ & 0.003 & 0.002 & 0.007 \\
\hline$\# E$ & 0.031 & 0.0020 & 0.0005 & 0.005 & $<0.001$ & 0.002 & 0.006 \\
\hline$\# F$ & 0.010 & 0.0012 & 0.0059 & 0.001 & $<0.001$ & 0.002 & 0.006 \\
\hline \#JM as-received & 0.004 & 0.0222 & 0.0042 & 0.001 & $<0.001$ & $<0.001$ & 0.002 \\
\hline
\end{tabular}

* Presented at the D. K. Chernov Centennial Symposium, May, 1968 in Moscow. Manuscript received September 9, 1968.

** Department of Metal Science and Technology, Kyoto University, Sakyo-ku, Kyoto 606.

*** Now at Research Laboratories, Kawasaki Steel Corp., Chiba 280.

**** Now at National Research Institute of Metals, Nakameguro, Meguro-ku, Tokyo 153. 
specimens thus purified was estimated to be less than 1 ppm by knowing the difference in the resistivities of specimens quenched from $300^{\circ} \mathrm{C}$ and fully-annealed, and also by adopting a reasonable value of the resistivity contribution of carbon or nitrogen of $2 \times 10^{-5} \Omega$ $\mathrm{cm} / \mathrm{wt}^{\mathrm{O}} / \mathrm{o}^{\mathrm{r})}$

Tensile tests were carried out on an Instron-type machine at a strain rate of $3 \times 10^{-4} \mathrm{sec}^{-1}$. Prior to straining, specimens were quenched from $500^{\circ}$ to $800^{\circ} \mathrm{C}$ into oil at $0^{\circ} \mathrm{C}$ in vacuum of about $10^{-4}$ torr, but specimens \#JM purified were tested without the quench. All resistivity measurements were carried out in liquid nitrogen, and the correction of the temperature of liquid nitrogen was made by using a dummy specimen. The accuracy of resistivity measurement thus obtained was about $1 \times 10^{-10} \Omega \mathrm{cm}$.

A special care was taken to determine the electrical resistivity of specimens after plastic deformation or heat treatments since there is some uncertainty to measure directly the size factor (length and diameter) of specimen. In such a case, the size factor for resistivity was determined by measuring the ratio of resistances of the specimen at two different temperatures such as $25^{\circ} \mathrm{C}$ and liquid nitrogen temperature, assuming Matthiessen's rule. It was also confirmed that Matthiessen's rule did approximately hold for iron containing carbon in the range of temperatures from liquid nitrogen to room temperature.

\section{Experimental Results}

\section{Annealing of Low Carbon Steels}

\section{(1) Below $400 \mathrm{C}$}

Figure 1 shows the change in electrical resistivity on $5 \mathrm{~min} / 20^{\circ} \mathrm{C}$ isochronal annealing for low carbon steel \# E $(0.03 \% \mathrm{G})$. Curve $I$ is the resistivity change of a specimen deformed in tension by $20 \%$ at $-75^{\circ} \mathrm{C}$ after the quench from $800^{\circ} \mathrm{C}$ into oil in vacuo, while curve $I I$ shows that of a specimen as-quenched but not deformed.

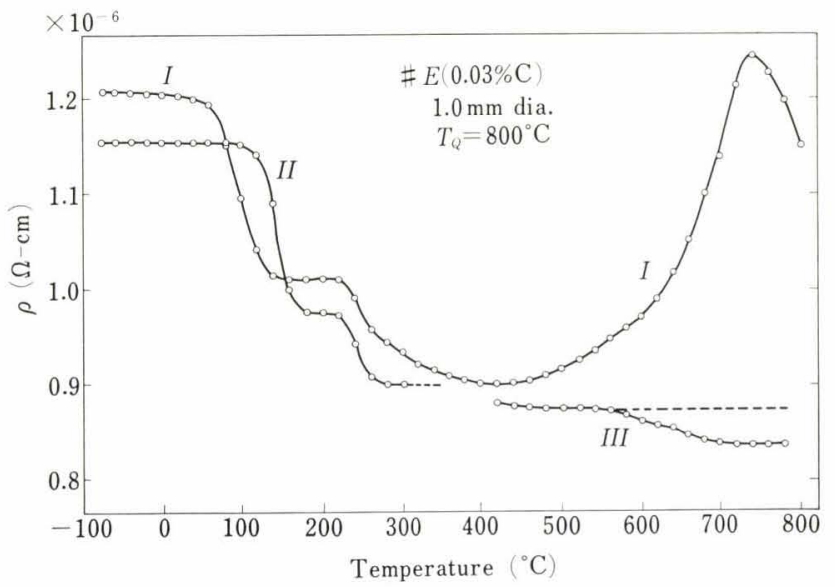

Fig. 1. The change in electrical resistivity on $5 \mathrm{~min} / 20^{\circ} \mathrm{C}$ isochronal annealing for specimen $\# E(0.03 \% \mathrm{C})$. Curve $I$ is for a specimen stretched by $20 \%$ at $-75^{\circ} \mathrm{C}$ after the quench from $800^{\circ} \mathrm{C}$, and curve $I I$ is for a specimen as quenched from $800^{\circ} \mathrm{C}$ but not deformed. Curve III was obtained by cooling slowly the same specimen as for curve $I$.
Each point on curve $I$ or $I I$ was obtained by measuring the resistivity of the specimen quenched from the isochronal annealing temperature.

Figure 2 shows the derivative curves for the data points below $400^{\circ} \mathrm{C}$ of Fig. 1. In an undeformed specimen (bottom curve in Fig. 2), two pronounced stages were found at about $160^{\circ}$ and $250^{\circ} \mathrm{C}$ as reported by Fujita and Damask. ${ }^{7}$ In a deformed specimen (top curve in Fig. 2), however, two additional stages were found around $-30^{\circ}$ and $300^{\circ} \mathrm{C}$ besides the two pronounced stages, also in good agreement with the observation by Fujita and Damask. It is to be noted that the central temperature of the most pronounced stage was shifted by plastic deformation from $160^{\circ}$ to $120^{\circ} \mathrm{C}$, while that of the stage around $250^{\circ} \mathrm{C}$ was not affected by straining.

Electron microscope observations indicated that the stages around $160^{\circ}$ and $250^{\circ} \mathrm{C}$ are related to the formation of metastable carbides and cementites, respectively, as generally accepted. ${ }^{8,9)}$ At the stage around $120^{\circ} \mathrm{C}$ for a deformed specimen finely dispersed particles of about $100 \mathrm{~A}$ were seen to have precipitated on strain-induced dislocations and also in the matrix, as shown in Photo. 1. The nature of the precipitates is not clear but could be carbon clustering or the pre-precipitation of fine carbides. It was noticed that the dislocation density in deformed specimens remained unchanged by annealing below $400^{\circ} \mathrm{C}$.

\section{(2) Above $400 \mathrm{C}$}

On isochronal annealing above $400^{\circ} \mathrm{C}$, the rapid increase in resistivity was observed because of the resolution of carbon into the matrix (curve $I$ in Fig. 1). Therefore, the stage corresponding to the annihilation

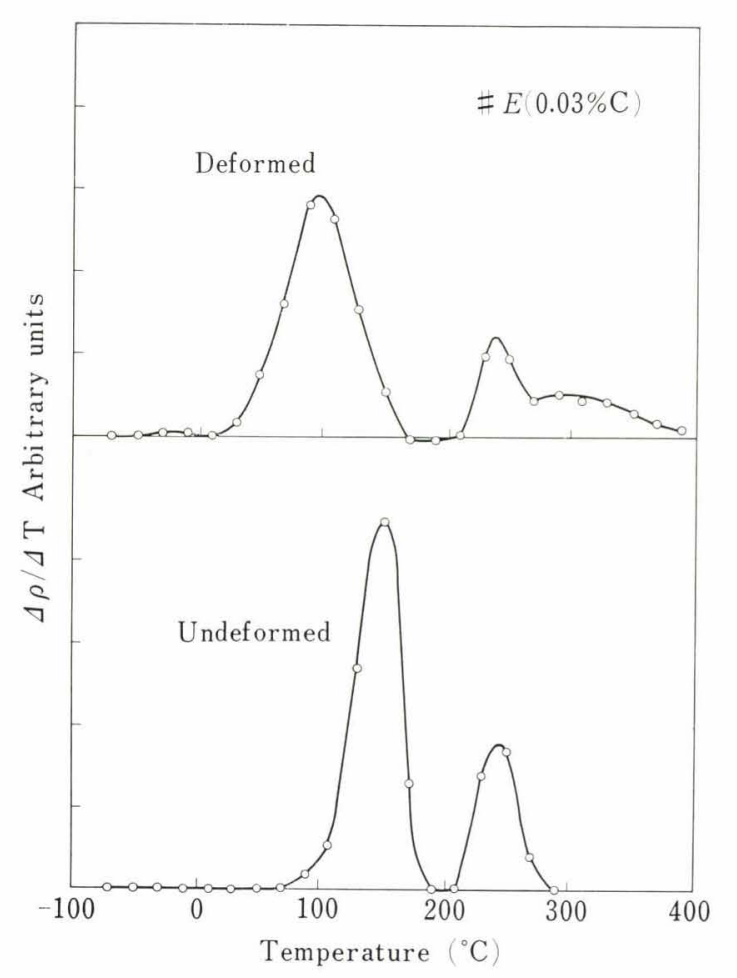

Fig. 2. The derivatives of isochronal curves $I$ and $I I$ below $400^{\circ} \mathrm{C}$ of Fig. 1 
of dislocations, even if it existed, could not be detected. In order to eliminate solute carbon from the matrix, specimens must be slowly cooled, rather than quenched, from the isochronal annealing temperature; it is only in this way that the stages can be observed. For convenience, specimens once quenched from isochronal annealing temperatures above $400^{\circ} \mathrm{C}$ were subjected to anneals at $400^{\circ} \mathrm{C}$ for $5 \mathrm{~min}$ and subsequently at $160^{\circ} \mathrm{C}$ for $5 \mathrm{~min}$, and then slowly cooled to room temperature. The result is shown in curve $I I I$ of Fig. 1 ; two distinct stages were first found around $600^{\circ}$ and $680^{\circ} \mathrm{C}$. From electron microscope observations it was made clear that the former stage was attributed to the rearrangement of dislocations such as the formation of sub-boundaries and the latter to the recrystallization process.

Lower yield stresses were measured at $25^{\circ} \mathrm{C}$ for specimens \#E and \#F after the quench from the annealing temperature, as shown in Fig. 3. Two stages were also observed exactly corresponding to those obtained from resistivity measurements, as clearly seen also in Fig. 4.

\section{Annealing of Pure Iron}

Figure 5 shows the annealing curves for the changes in electrical resistivity and yield stress for pure iron (\#JM purified) deformed by $20 \%$ at $25^{\circ} \mathrm{C}$. The specimens were annealed in dry hydrogen at each temperature and slowly cooled to room temperature. When the hydrogen atmosphere was not used for annealing, the increase in resistivity, instead of the decrease, was observed above $550^{\circ} \mathrm{C}$, presumably because of the contamination from diffusion-pump oil.

On $5 \mathrm{~min} / 20^{\circ} \mathrm{C}$ isochronal annealing from room temperature to $400^{\circ} \mathrm{C}$ the recovery of strain-induced resistivity was observed in a rather continuous way without pronounced stages as reported by Cuddy, ${ }^{2}$ whereas no change in yield stress was observed up to about $400^{\circ} \mathrm{C}$ as clearly seen in the bottom curve of Fig. 3. Above $400^{\circ} \mathrm{C}$, however, two recovery stages were detected for both electrical resistivity and yield stress similarly to those for low carbon steels, as understood from Fig. 5 ; the less-pronounced first stage is

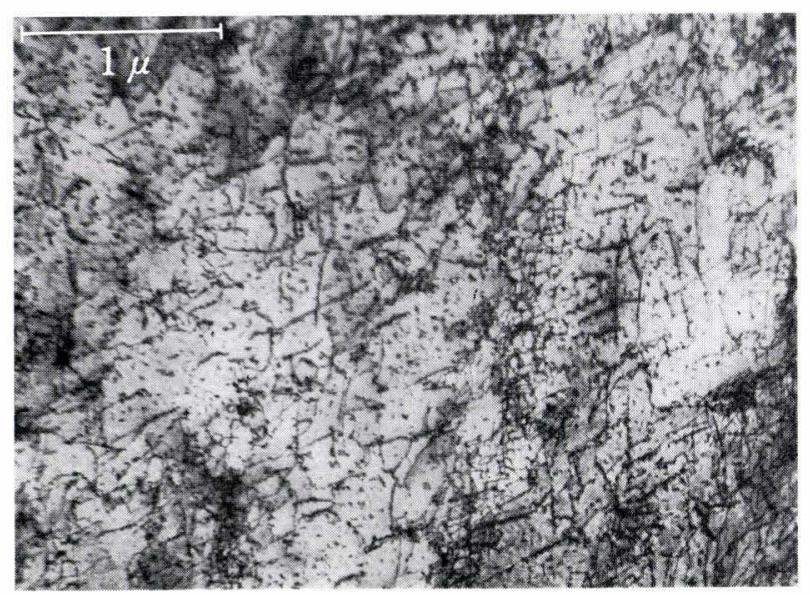

Photo. 1. Precipitates observed on annealing at $120^{\circ} \mathrm{C}$ in low carbon steel \#E $(0.03 \% \mathrm{C})$, which was quenched from $800^{\circ} \mathrm{C}$ and deformed by $20 \%$ at $-75^{\circ} \mathrm{C}$. centered around $480^{\circ} \mathrm{C}$ and the distinct second stage at $550^{\circ} \mathrm{C}$.

\section{Effects of Solute Carbon and Grain Size on the Strain- Induced Resistivity}

In order to change the solute carbon content specimens $\# \mathrm{~A}, \# \mathrm{E}$, and $\# \mathrm{~F}$ were quenched from various temperatures, and the resistivity increment was measured after deformation by $20 \%$ at $-75^{\circ} \mathrm{C}$. The results are shown in Fig. 6. The abscissa of Fig. 6 is the resistivity ratio $\left(\rho_{298} / \rho_{77}\right)$ as a measure of the

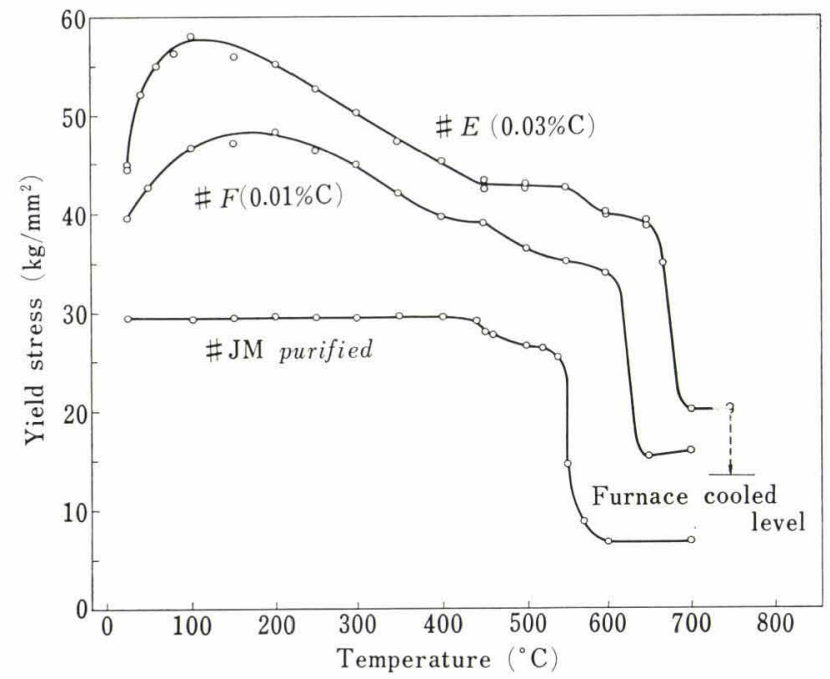

Fig. 3. Lower yield stresses measured at $25^{\circ} \mathrm{C}$ for specimens \#E $(0.03 \% \mathrm{C}), \# F \quad(0.01 \% \mathrm{C})$ and \#JM purified. Specimens $\# E$ and $\# F$ were quenched from the annealing temperature, while specimen \#JM purified was furnace cooled. Specimens $\# E$ and $\# F$ were stretched by $20 \%$ at $-75^{\circ} \mathrm{C}$ and specimen \#JM purified was deformed by $20 \%$ at room temperature, prior to the annealing for $5 \mathrm{~min}$ at each temperature.

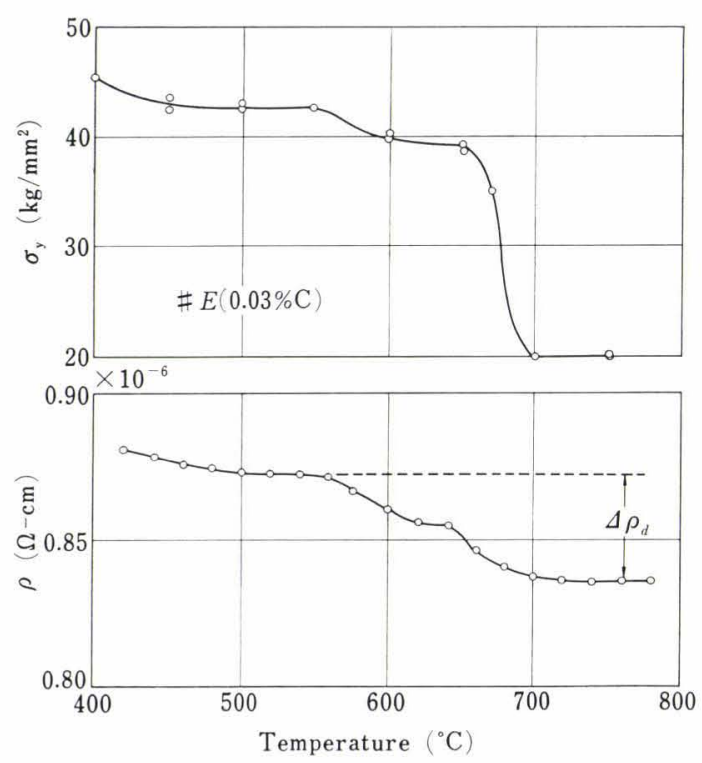

Fig. 4. Changes in lower yield stress and electrical resistivity on annealing at temperatures above $400^{\circ} \mathrm{C}$ for specimen \#E $(0.03 \% \mathrm{C})$ 
content of solute carbon. Specimens \#E and \#F in Fig. 6 show that the resistivity increment due to plastic deformation increases with increasing the quench temperature, namely, with increasing the content of solute carbon, in good agreement with the observation by Thomas and Leak. ${ }^{10)}$ However, specimens \#A, regardless of quenching temperatures over the range

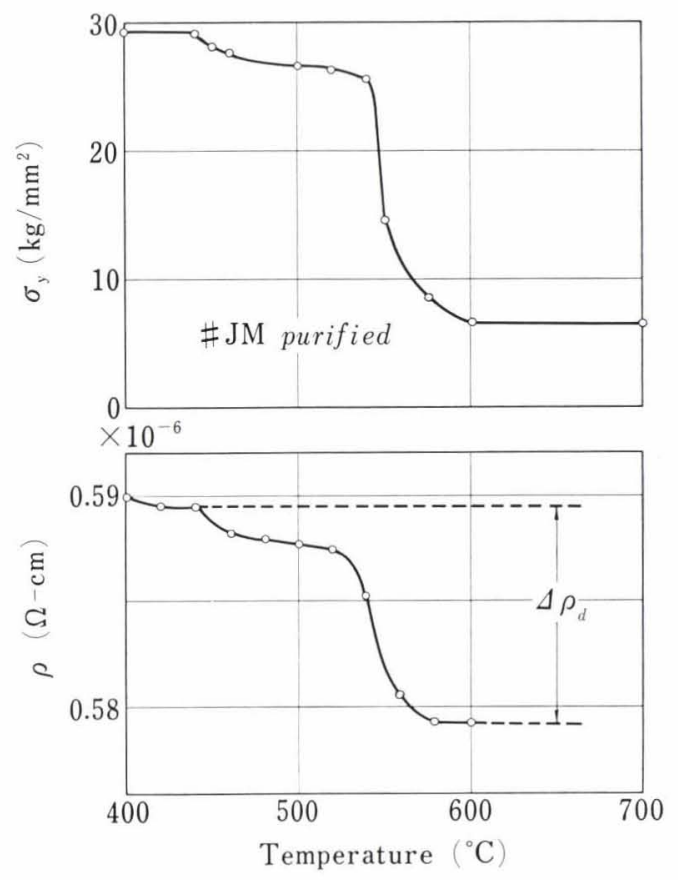

Fig. 5. Changes in yield stress and electrical resistivity on isochronal annealing at temperatures above $400 \mathrm{C}$ for specimen \#JM purified

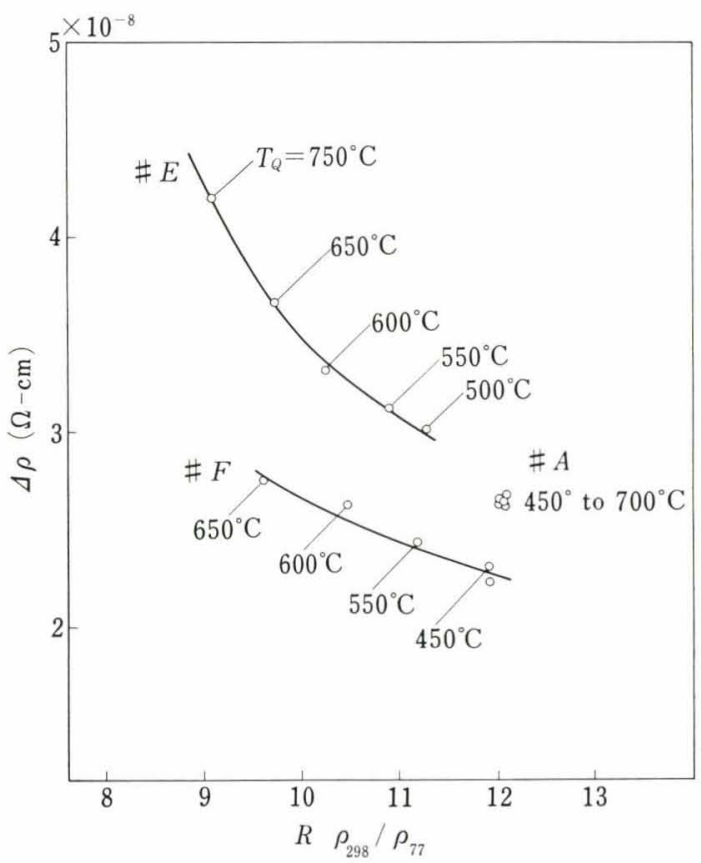

Fig. 6. The resistivity increment for specimens \#A $(0.002 \% \mathrm{C})$, \#E $(0.03 \% \mathrm{C})$, and \#F $(0.01 \% \mathrm{C})$ stretched by $20 \%$ at $-75^{\circ} \mathrm{C}$ after the quench from various temperatures. The abscissa is the resistivity ratio, $R=\rho_{298} / \rho_{77}$, which is an indication of the solute content. from $450^{\circ}$ to $700^{\circ} \mathrm{C}$, had almost the same value of the resistivity increment as well as the resistivity ratio. This indicates that all carbon $(20 \mathrm{ppm})$ are in solution at temperatures above $450^{\circ} \mathrm{C}$, and hence the amount of lattice defects produced by deformation is almost unchanged even by varying the quench temperature.

The amount of lattice defects produced by deformation is also affected by the grain size of specimen, as pointed out by Keh and Weissmann ${ }^{11)}$ and Cuddy. ${ }^{2}$ The grain sizes of specimens \#E and \#F in Fig. 6 are 8 and $14 \mu$, respectively. Therefore, the difference in resistivity increments between specimens \#E and \#F for a given value of resistivity ratio $\rho_{298} / \rho_{77}$ should be ascribed to the difference in the grain size. To confirm this effect, specimens \#F $(0.01 \% \mathrm{C})$ having the grain size of 13 to $30 \mu$ were prepared and stretched by $20 \%$ at $-75^{\circ} \mathrm{C}$ after the quench from $550^{\circ}$ and $650^{\circ} \mathrm{C}$. The grain-size control was made by annealing the specimen at temperatures above $700^{\circ} \mathrm{C}$. The results for resistivity measurements are shown in Fig. 7 ; the total resistivity increment is found to be inversely proportional to grain diameter $d$ as

$$
\Delta \rho(\Omega \mathrm{cm})=2.1 \times 10^{-8}+9.4 \times 10^{-8} d^{-1}
$$

for the quench from $650^{\circ} \mathrm{C}$,

$$
\Delta_{\rho}(\Omega \mathrm{cm})=2.0 \times 10^{-8}+5.9 \times 10^{-8} d^{-1}
$$

for the quench from $550^{\circ} \mathrm{C}$,

where $d$ is in microns. The results show that the coefficient of the second term, i.e., the grain size dependence on the production of lattice defects is affected by the carbon content in solution. However, it is to be noted that the content of solute carbon to be retained in the matrix by the solution treatment, when measured from electrical resistivity, is decreased as the grain size of specimen is reduced. Therefore, the above values of the first and second terms must be somewhat corrected in the strict sense.

\section{Discussion}

From resistivity measurements on isochronal annealing of deformed low carbon steels, two stages due to the annihilation of dislocations were found at temperatures above $400^{\circ} \mathrm{C}$, exactly corresponding to the stages obtained from the measurement of yield stresses

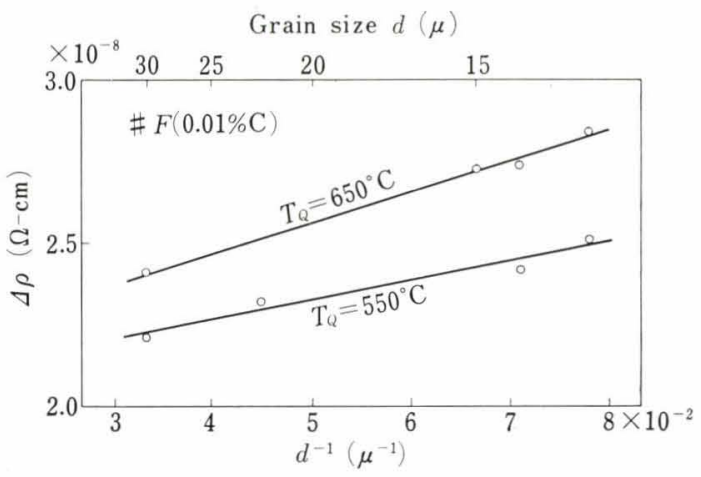

Fig. 7. Relation between the grain size of specimen and the strain-induced resistivity increment for specimens $\# F$ $(0.01 \% \mathrm{C})$ 
(Figs. 1, 3, and 4). The reason why these stages have not been so far reported may be due to the lack of controlling the cooling rate from the isochronal annealing temperature. The distinct stages were first observed in the present investigation only by the careful heat treatments so performed as to eliminate solute carbon from the matrix.

In pure iron the two stages were also found, when annealed in dry hydrogen to prevent possible contamination by air and diffusion-pump oil (Figs. 3 and 5). Keh ${ }^{12}$ ) showed from the direct observation of deformed pure iron that there was little change in dislocation arrangements even after annealing for $1 \mathrm{hr}$ at $400^{\circ} \mathrm{C}$ or below, and that the significant change was first detected above $500^{\circ} \mathrm{C}$. This observation is in good agreement with the present results shown in the bottom curve of Fig. 3 and in Fig. 5, in which no change of yield stress is seen up to $440^{\circ} \mathrm{C}$.

On the other hand, Cuddy ${ }^{2)}$ has pointed out from the resistivity measurements of pure iron that dislocations can rearrange themselves at temperatures far below $400^{\circ} \mathrm{C}$ and even at room temperature, since there was no indication of any uniquely activated process other than a continuous recovery up to $500^{\circ} \mathrm{C}$. In the present case a rather continuous recovery of resistivity was also observed over the entire range from room temperature to $440^{\circ} \mathrm{C}$ on isochronal annealing of pure iron (\#JM purified) stretched by $20 \%$ at $25^{\circ} \mathrm{C}$. However, as seen in Fig. 5, the continuous recovery of resistivity is followed by the well-defined two stages due to the annihilation of dislocations, which exactly correspond to the stages for yield stress. The amount of resistivity recovery up to $440^{\circ} \mathrm{C}$ is $1 \times 10^{-8} \Omega \mathrm{cm}$, which is almost exactly one half of the total resistivity increment due to deformation. If the elimination of interstitial impurities from the matrix is responsible for the continuous recovery up to $440^{\circ} \mathrm{C}$, the decrement is expected to be at most $2 \times 10^{-9} \Omega \mathrm{cm}$, even if all the interstitial impurities ( $<1 \mathrm{ppm})$ is assumed to become non-contributory to resistivity. Thus the resistivity recovery up to $440^{\circ} \mathrm{C}$, at least in pure irons studied, is mainly attributable to the annihilation of point defects produced by plastic deformation.

The amount of lattice defects introduced by plastic deformation can be determined by knowing the resistivity contribution of defects, provided that the total resistivity increment by deformation and the resistivity change due to the annihilation of dislocations are known. The resistivity change due to the annihilation of dislocations is determined experimentally as $\Delta \rho_{d}$ which is indicated in Figs. 4 and 5 . Typical results are listed in Table 2 for specimens \#E $(0.03 \%$ C) deformed by $20 \%$ at $-75^{\circ} \mathrm{C}$ and for pure iron deformed by $20 \%$ at $25^{\circ} \mathrm{C}$. The difference between $\Delta \rho$ (total increment) and $\Delta \rho_{d}$ should represent the resistivity change due to point defects produced by plastic deformation. If values of $1 \times 10^{-18} \Omega \mathrm{cm}^{3}{ }^{2), 13)}$ and $2.5 \times 10^{-6} \Omega \mathrm{cm}^{7}$ ) are adopted as the resistivity contribution per unit density of dislocations and atomic per cent of point defects, respectively, the amount of lattice defects is estimated as shown in Table 2. The
Table 2. The strain-induced resistivity and the amount of lattice defects in deformed low carbon steels and pure iron

\begin{tabular}{c|cc|c|c}
\hline Specimen & \# E $(0.03 \% \mathrm{C})$ & $\begin{array}{c}\text { \#.JM } \\
\text { purified }\end{array}$ & \\
\cline { 1 - 4 } Quench temp. & $800^{\circ} \mathrm{C}$ & $700^{\circ} \mathrm{C}$ & & \\
\hline$\Delta_{\rho}$ (total) & 4.8 & 3.8 & 2.0 & $\times 10^{-8} \Omega \mathrm{cm}$ \\
\hline$\Delta_{\rho_{d} \text { (disloc.) }}$ & 3.7 & 2.8 & 1.0 & $\times 10^{-8} \Omega \mathrm{cm}$ \\
\hline$\Delta_{\rho}-\Delta_{\rho_{d}}$ & 1.1 & 1.0 & 1.0 & $\times 10^{-8} \Omega \mathrm{cm}$ \\
\hline Dislocation & 3.7 & 2.8 & 1.0 & $\times 10^{10} \mathrm{~cm}^{-2}$ \\
\hline Point defect & 4.4 & 4.0 & 4.0 & $\times 10^{-5}$ \\
\hline
\end{tabular}

dislocation density and the concentration of point defects were found to be of the order of $10^{10} \mathrm{~cm}^{-2}$ and $10^{-5}$, respectively.

One thing that must be considered for the determination of the defect population in low carbon steels is the change in the contribution to resistivity of solute carbon atoms when attached to dislocations. At annealing temperatures above $500^{\circ} \mathrm{C}$ most carbon atoms are free from dislocations, but during quenching from the annealing temperatures they can be trapped by dislocations because of the strong interaction (needless to say, become more trapped when slowly cooled). The resistivity contribution of such attached carbon atoms is not accurately known, but it could be fairly smaller than that of free carbon atoms in the matrix, as expected from the nature of electron scattering. If this is the case, the difference in the resistivity contributions between free and trapped carbon atoms must be added to the value of $\Delta \rho_{d}$ in Table 2 . However, the change in resistivity due to such effect will not exceed $15 \%$ of the measured values of $\Delta \rho_{d}$, even if the resistivity contribution of these attached carbon atoms is entirely ignored and also all dislocations before annihilation are assumed to have, at every atomic distance, been decorated with carbon atoms.

From Table 2 it is noticed that in pure iron the strain-induced resistivities due to dislocations and point defects are almost equal, whereas in low carbon steels the strain-induced resistivity due to dislocations is larger than that for point defects. This fact suggests that solute carbon atoms are very effective to produce or retain more dislocations in the specimen, giving rise to the increase in flow stress. The amount of point defects in different specimens is almost the same as seen in Table 2 ; the reason is not clear yet, but this might be incidental.

\section{Summary}

The strain-induced resistivity and the annealing stage were investigated for low carbon steels deformed in tension by $20 \%$ at $-75^{\circ} \mathrm{C}$ and also for high purity iron deformed by $20 \%$ at $25^{\circ} \mathrm{C}$. The main results are as follows.

(1) Successive two distinct annealing stages corresponding to the annihilation of dislocations were found 
for the first time by electrical resistivity measurements not only in low carbon steels but also in high purity iron (Figs. 3, 4, and 5). The two stages are associated with the formation of sub-boundaries and the recrystallization. The fact was confirmed by the measurement of flow stresses and also by transmission electron microscopy.

(2) At temperatures below $400^{\circ} \mathrm{C}$, dislocations introduced by plastic deformation are scarcely annihilated even in high purity iron (at least in specimens studied) on $5 \mathrm{~min} / 20^{\circ} \mathrm{C}$ isochronal annealing (see the curve of \#JM purified in Fig. 3).

(3) On annealing below $400^{\circ} \mathrm{C}$ of deformed low carbon steels, recovery stages were found around $-30^{\circ}$ and $300^{\circ} \mathrm{C}$ in addition to the two pronounced stages observed in undeformed specimens (Fig. 2).

(4) The amount of lattice defects introduced by plastic deformation was determined from the resistivity change due to the annihilation of dislocations and the total strain-induced resistivity and by adopting the reasonable value for the resistivity contribution of lattice defects. The dislocation density and concentration of point defects were of the order of $10^{10} \mathrm{~cm}^{-2}$ and $10^{-5}$, respectively, in specimens deformed by $20 \%$ (Table 2). It was also observed that strain-induced resistivities due to dislocations and point defects are almost equal in high purity iron.

(5) The amount of lattice defects, and in particular the density of dislocations, increases as the content of solute carbon is increased and also as the grain size of specimen is reduced (Figs. 6 and 7). It is suggested that solute carbon atoms are very efficient to produce or retain more dislocations in a specimen, giving rise to the increase in flow stress.

\section{Acknowledgements}

The authors wish to thank Mr. K. Ono for his assistance in the experimental work and are also grateful to the Iron and Steel Institute of Japan for the financial support to a part of this work.

\section{REFERENCES}

1) T. Taoka, K. Suzuki, A. Yoshikawa, and M. Okamoto: Acta Met., 13 (1965), 1311.

2) L. J. Cuddy: Phil. Mag., 12 (1965), 855.

3) T. Oi and K. Sato: Trans. Japan Inst. Metals, 7 (1966), 32.

4) A. Yoshikawa and M. Okamoto: J. Phys. Soc. Japan, 22 (1967), 996.

5) For example, A. R. Rosenfield: Acta Met., 12 (1964), 119.

6) L. Berger and A. R. de Vroomen: J. Appl. Phys., 36 (1965), 2777.

7) F. E. Fujita and A. C. Damask: Acta Met., 12 (1964), 331.

8) W. G. Leslie: Acta Met., 9 (1961), 1004.

9) J.F. Butler: J. Iron and Steel Inst., 204 (1966), 127.

10) W. R. Thomas and G. M. Leak: J. Iron and Steel Inst., 180 (1955), 155.

11) A. S. Keh and S. Weissmann: Electron Microsocopy and Strength of Crystals, (1963), 231, Interscience, New York.

12) A. S. Keh: Direct Observation of Imperfections in Crystals, (1962), 213, Interscience, New York.

13) Z. S. Basinski, J. S. Dugdale, and A. Howie: Phil. Mag., 8 (1963), 989. 